\title{
Posneoliberalismo y después: el Centro Relativo del Sistema POLÍTICO Y EL ASCENSO DE LA MAREA CELESTE EN EL ESPEJO DE LA política exterior de Argentina y Chile
}

\author{
Post-Neoliberalism and After: The Relative Center of the Political \\ System and the Rise of the Light Blue Wave in the Mirror of the Foreign \\ Policy of Argentina and Chile
}

\section{MARÍA ELENA LORENZINI}

Consejo Nacional de Investigaciones Científicas y Técnicas, Universidad Nacional de Rosario, Argentina

\section{GISELA PEREYRA DOVAL}

Consejo Nacional de Investigaciones Científicas y Técnicas, Universidad Nacional de Rosario, Argentina

\begin{abstract}
RESUMEN
El objetivo del artículo es interpelar los giros a la izquierda ocurridos en la primera década del siglo xxi y, a la derecha, en la segunda. Para ello aplicamos el abordaje utilizado por Coppedge $(1997,2000)$ y construimos un mapa político de Sudamérica. El vehículo empírico seleccionado es la política exterior de Argentina y Chile, cuyo análisis nos permite corroborar, o no, los desplazamientos del centro relativo del sistema político (CRSP). Así, hallamos que el sistema político regional se ubicó en una posición de centro, mostrando un sobredimensionamiento de los giros políticos en la producción académica reciente. En los sistemas políticos más estables, la política exterior no mostró cambios, mientras que en aquellos sistemas que atravesaron ciclos de signo diverso, la política exterior experimentó modificaciones de diversa intensidad.
\end{abstract}

Palabras clave: giros políticos, izquierda, derecha, centro relativo del sistema político, política exterior, Sudamérica

\begin{abstract}
This article questions the political turns to the left in the first decade of the 21st century and to the right in the second. To do this, we build a political map of South America, applying Coppedge's $(1997,2000)$ approach to determine the "relative center of the political system" (RCPS). We focus on Argentina's and Chile's foreign policy to corroborate the displacements or not of the RCPS. We find that both countries gravitated towards the center, indicating an overemphasis on the political turns in recent academic writing. In the most stable political systems, foreign policy did not change, while in those systems that went through diverse cycles, foreign policy changed in varying degrees.
\end{abstract}

Keywords: Political turn, Left, Right, Relative Center of the Political System, Foreign Policy, South America 


\section{INTRODUCCIÓN}

Durante la primera década del siglo xxi proliferó bibliografía que problematizó un nuevo fenómeno político en Sudamérica: las características de los procesos políticos a partir del ciclo electoral regional iniciado en 1998 con el triunfo de Hugo Chávez en Venezuela. En adelante, los académicos caracterizaron los procesos políticos como "giro a la izquierda", "progresismo", "ascenso de la marea rosa", "posneoliberalismo", "neopopulismo", etcétera. El común denominador de dichos trabajos daba cuenta de los ciclos políticos sudamericanos a la luz de los resultados de las elecciones realizadas en el ocaso de la década neoliberal. La polémica surgió con la publicación del artículo de Castañeda (2006) en el que se clasificaba la izquierda en dos grupos: moderada, representada por Bachelet, Tabaré Vázquez y Lula da Silva, y populista, representada por Kirchner, Chávez, Correa y Morales.

Una década después, el debate cobró nuevos bríos en la academia y en la política sudamericana con los resultados de los ciclos electorales, cuyos triunfadores fueron líderes etiquetados de derecha. Por tal motivo, los académicos debaten sobre el denominado "giro a la derecha". Hablar de "giros" responde a que la noción introduce matices de gradualidad en términos comparados con otras categorizaciones y, al mismo tiempo, es compatible con el argumento de Coppedge $(1997,2000)$ sobre el centro relativo del sistema político (CRSP) ${ }^{1}$, orientado a eludir los reduccionismos convencionales izquierda-derecha, pues incorpora categorías relativas. Los giros implican cierto grado de movimiento en torno a un centro o eje, mostrando un recorrido entre un punto de partida $\mathrm{y}$ un punto de llegada, pudiendo ser graduales o repentinos. El movimiento alude al dinamismo y la dirección que pueden adquirir las posiciones internas y externas de un gobierno sobre temas y actores en contextos específicos. En esa dirección, Panizza (2006) afirmó que el cuadro electoral de comienzos de siglo mostró el advenimiento de una marea rosa y sostuvo que el color político de los gobiernos regionales fue modificándose a medida que los procesos electorales transcurrían. Para ilustrar los procesos políticos en la segunda década del siglo XXI, construimos la categoría de marea celeste. La utilización del rosa, en lugar del rojo, y del celeste, en lugar del azul, trasmite la idea de matices que, como los pigmentos, pueden ser más o menos intensos. Además, rosa y celeste se asocian con la existencia de distintos grados e intensidades, aproximándose a lo relativo y apartándose de lo absoluto.

Este trabajo se diferencia del de Coppedge respecto del objetivo que lo orienta. Su foco era estudiar los sistemas de partidos en Latinoamérica, mientras que aquí analizamos los giros políticos sudamericanos. Para catalogar los competidores de cada elección presidencial, combinamos las orientaciones políticas de los candidatos - expresadas en sus discursos-y las orientaciones políticas generales de los partidos políticos que representaban. Esto ayuda a comprender, por ejemplo, los cambios de cuadrantes de los candidatos del Partido Justicialista en Argentina. Otra diferencia es que en el análisis de elecciones legislativas observó la fragmentación y polarización de los SP. En nuestro caso, analizamos los giros políticos en ciclos electorales distintos: marea rosa y celeste. 
En este contexto, revisamos el planteo de Coppedge acerca del CRSP, siendo nuestro objetivo central interpelar los giros ideológicos, aplicando la metodología utilizada por este autor, para construir un mapa político de Sudamérica desde la primera década del siglo xxI hasta 2015. Para alcanzarlo, analizamos los cambios de signo político en los gobiernos y examinamos si estos se reflejaron en la política exterior (PE) como vehículo empírico. Ella, siguiendo a Clemente Batalla (2007), es la resultante de complejas articulaciones entre factores internos ${ }^{2}$ y externos que operan en un contexto de cambio constante de acuerdo con la coyuntura, modificando las opciones posibles de inserción internacional.

Tomamos como referencia un conjunto de dimensiones que nos permiten relevar la orientación general de la PE a partir de la ubicación del cRsP. Ellas son: prioridades geográficas norte-sur, multilateralismo statuquoista versus multilateralismo revisionista light y regionalismo económico laxo versus regionalismo económico estricto.

Para dar cuenta del objetivo, explicamos el CRSP y aplicamos este índice en las elecciones presidenciales en diez países sudamericanos ocurridas entre 1998 y noviembre $2015^{3}$, para determinar hacia qué cuadrantes se movió: derecha, centroderecha, centro, centroizquierda o izquierda. En esta instancia, identificamos las dificultades encontradas y el modo a través del cual decidimos sortearlas. En segundo lugar, escogimos los casos argentino y chileno, pues ilustran empíricamente cómo los giros políticos se reflejaron en los ejes seleccionados de PE. Este trabajo aspira a contribuir a ese debate a partir del estudio de los aportes que la lectura del CRSP nos permite realizar.

Los estudios sobre cambios y continuidades en PE son un tema recurrente y de larga data en relaciones internacionales. El discurso de Hermann, como presidente de la International Studies Association en 1989, constituyó un hito, subrayando las variables sistémicas y domésticas relativas al cambio. Desde el punto de vista doméstico, Hermann y Hermann (1989), Rosenau (1995) y Hermann (2001) pusieron el acento en los procesos de toma de decisiones. En el mismo nivel se estudiaron los factores psicológicos de la personalidad de los líderes (George 1993; Hudson 2005), el impacto del cambio de régimen político (Holsti 1982; Doyle 1986), el rol de las ideologías (Goldstein y Keohane 1993; Rosatti 2000) y los modelos de desarrollo (Curia 2011; Actis et al. 2016; Pereyra Doval 2016). Desde el punto de vista sistémico, se identificó un conjunto de

2 La PE refleja principios, creencias, valores, intereses y necesidades de un gobierno, provenientes del sistema político y del modelo de desarrollo, conjugando elementos originados en el conjunto de políticas públicas económicas, sociales y financieras (Busso 2016). En este punto, anticipamos la idea que la PE cambia en mayor medida porque cambian las configuraciones y los contenidos de las políticas domésticas, sin descartar la incidencia de factores externos. No obstante, existen PE más estables en países menos vulnerables que los latinoamericanos.

3 Nos permite incorporar los resultados de las elecciones venezolanas de 1998 - las primeras en las que ascendió un líder de la marea rosa - hasta la primera vuelta electoral en Argentina como punto de partida del ascenso de la marea celeste. En este cuadro de situación sobresalen algunas medidas gubernamentales que van a contrapelo de la marea del momento. Un ejemplo es el giro ortodoxo implementado por Dilma Rousseff 2013-2014. 
variables para explicar el cambio en la PE (Risse-Kappen 1994; Doeser 2011), configuración del orden mundial, polaridad (Waltz 1988, 2000), distribución de atributos materiales (Schmidt 2008; Wohlforth 2008) e institucionalización del sistema internacional (Keohane 1988; Grasa 1993).

Acorde con ello, la hipótesis sostiene que los cambios en los CRSP, medidos por resultados de elecciones presidenciales, tienen efectos en las alteraciones de la PE. El criterio de selección de los casos se basa en que Argentina experimentó giros políticos en sus elecciones presidenciales cuyo derrotero fue centroizquierda/centroderecha/centroizquierda del CRSP. En Chile, pese a los cambios de gobierno, el sistema político (sP) se mantuvo dentro del centro relativo. Ambos casos resultan paradigmáticos: Argentina fue el único país sudamericano que experimentó giros políticos en cuadrantes ideológicos opuestos que fueron acompañados de cambios en $\mathrm{PE}^{4}$, mientras que Chile fue el único país sudamericano que permaneció en el CRSP durante el período 19982015 y su PE conservó un alto grado de continuidad. Pese a que los ocho países restantes experimentaron giros, sus trayectorias no mostraron desplazamientos hacia cuadrantes ideológicos opuestos.

\section{UBICACIÓN DEL CRSP EN SUDAMÉRICA (1998-2015)}

Coppedge $(1997,2000)$ define el CRSP como un índice que mide la distancia hacia la izquierda o hacia la derecha en la que se ubican los partidos promedios de los países en cada elección, basándose en la posición izquierda-derecha de todos los competidores y utilizando los porcentajes de votos obtenidos. Así, el CRSP puede estar más inclinado hacia la derecha o hacia la izquierda del centro absoluto. Un primer punto importante es que los SP orbitan en torno al centro. Esto significa que, en Sudamérica, los presidentes se posicionan o son posicionados a la izquierda o a la derecha del sp cuando, en realidad, circulan en torno al centro y solo mediante algunas medidas específicas "giran" hacia el lado contrario.

No obstante las lagunas encontradas, consideramos que el planteo sobre el CRSP y sus fundamentos son útiles para observar los giros en la política sudamericana. Por tales motivos, lo aplicamos en las elecciones del Poder Ejecutivo $^{5}$ sudamericanas, partiendo de la elección presidencial más lejana en la que triunfó un líder asociado al ascenso de la marea rosa - Hugo Chávez (1998) — hasta la última elección presidencial argentina - Mauricio Macri (2015) - , que coincide con el momento en el que diversos autores advierten un giro a la derecha (Giordano 2014; O’Neil 2016). 
En segundo lugar, siguiendo a Coppedge $(1997,2000)$, clasificamos los candidatos presidenciales que compitieron en cada elección en derecha, centroderecha, izquierda y centroizquierda, entre 1998 y 2015 en Argentina, Bolivia, Brasil, Colombia, Chile, Ecuador, Paraguay, Perú, Uruguay y Venezuela. Además, tomamos el porcentaje de votos obtenido por cada candidato. Cabe aclarar que excluimos las segundas vueltas ${ }^{6}$. Esta es la primera laguna hallada en la construcción del índice, pues restringe el universo de expresiones políticas que participan y polariza el espectro. En el caso de haberlas incluido, se hubiera generado una distorsión para calcular los promedios, puesto que no en todos los países - tampoco en todas las elecciones- hubo balotaje.

En tercer lugar, aplicamos la fórmula para medir el centro relativo de cada SP sudamericano, que se expresa:

$(\%$ votos derecha $+0,5 \%$ votos centro-derecha $)-(0,5 \%$ de votos centro-izquierda $\%$ votos izquierda)

Al aplicar la fórmula, obtuvimos los índices que muestran la ubicación del CRSP por país (tabla 1). Asimismo, establecimos un rango +41 y -41 para describir la inclinación hacia la derecha/centroderecha/centro relativo/centroizquierda/ izquierda de cada sp. Los SP entre (+ 21 y 40) se ubican a la centroderecha del CRSP; mayores a 41, a la derecha. Entre (-21 y -40) se ubican en la centroizquierda y mayor a -41, en la izquierda. En los casos en los que los índices alcanzan un valor entre -20 y 20, se localizan en el cRsP (gráfico 1 y tabla 2).

Tabla 1. CRSP por país por elección período completo

\begin{tabular}{|c|c|c|c|c|c|c|c|c|c|c|}
\hline & 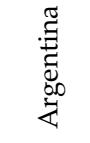 & 氶 & 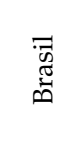 & $\stackrel{\mathscr{J}}{\stackrel{\Xi}{\Xi}}$ & $\begin{array}{l}\frac{\pi}{0} \\
\frac{0}{0} \\
\frac{0}{0} \\
0\end{array}$ & 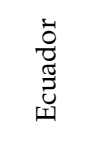 & 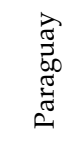 & 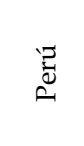 & $\begin{array}{l}\vec{J} \\
\text { ప్ర } \\
\vec{J}\end{array}$ & 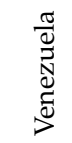 \\
\hline 1998 & & & 1,85 & & 14,19 & 37,65 & 33,45 & & & $-8,12$ \\
\hline 1999 & $-36,1$ & & & $-3,41$ & & & & & 4,87 & \\
\hline 2000 & & & & & & & & 37,46 & & $-10,3$ \\
\hline 2001 & & & & & & & & $-3,97$ & & \\
\hline 2002 & & 15,25 & $-41,5$ & & 35,97 & 37,11 & & & & \\
\hline 2003 & 21,71 & & & & & & 30,35 & & & \\
\hline 2004 & & & & & & & & & $-2,89$ & \\
\hline 2005 & & $-15,6$ & & $-4,06$ & & & & & & \\
\hline
\end{tabular}

Coppedge $(1997,2000)$ construye otro indicador para medir la polarización: MLRP/100. El MLRP (mean left right position) es el índice de la posición ideológica promedio que mide la distancia izquierda-derecha del partido promedio en cada elección, tomando como referencia las posiciones izquierda-derecha de todos los partidos vía porcentaje de votos. 


\begin{tabular}{|c|c|c|c|c|c|c|c|c|c|c|}
\hline & 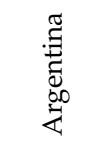 & 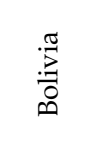 & 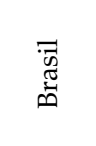 & 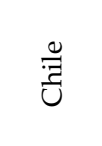 & $\begin{array}{l}\frac{\pi}{0} \\
\text { है } \\
\frac{0}{0} \\
0\end{array}$ & $\frac{\dot{0}}{0}$ & $\begin{array}{l}\vec{\Xi} \\
\vec{J} \\
\mathbb{\Xi} \\
\vec{J} \\
0\end{array}$ & ב."ב & $\begin{array}{l}\overrightarrow{0} \\
\overrightarrow{0} \\
\overrightarrow{5} \\
\overrightarrow{5} \\
5\end{array}$ & 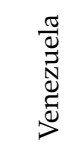 \\
\hline 2006 & & & $-11,94$ & & 43,61 & 13,95 & & $-11,1$ & & $-12,97$ \\
\hline 2007 & $-40,4$ & & & & & & & & & \\
\hline 2008 & & & & & & & 19,14 & & & \\
\hline 2009 & & $-17,43$ & & $-3,36$ & 45,41 & 4,58 & & & $-2,39$ & \\
\hline 2010 & & & $-16,82$ & & & & & & & \\
\hline 2011 & $-43,17$ & & & & & & & 17,54 & & \\
\hline 2012 & & & & & & $-8,41$ & & & & $-5,38$ \\
\hline 2013 & & & & $-12,69$ & 45,09 & & $-0,24$ & & & $-0,74$ \\
\hline 2014 & & $-16,76$ & $-16,23$ & & & & & & $-2,59$ & \\
\hline 2015 & 5,57 & & & & & & & & & \\
\hline
\end{tabular}

Fuente: elaboración propia a partir de los datos oficiales obtenidos de las instituciones electorales de cada país

\section{Gráfico 1. MLRP: 45 elecciones presidenciales (1998-2015)}

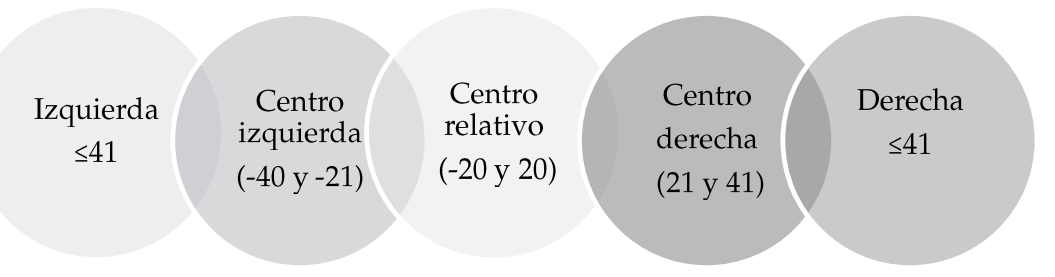

Fuente: elaboración propia

(I): Brasil 2002

(CI): Argentina 1999, 2007, 2011

(CR): Argentina (2015); Bolivia (2002, 2006, 2009, 2014); Brasil (1998, 2006, 2010, 2014); Ecuador (2006, 2009, 2012); Paraguay (2006, 2013); Perú (2001, 2006, 2011); Uruguay (1999, 2004, 2009, 2014); Venezuela (1998, 2000, 2006, 2012, 2013)

(CD): Argentina (2003); Colombia (2002); Ecuador (1998, 2003); Paraguay (1998, 2003); Perú (2000)

(D): Colombia $(2006,2009,2014)$

A partir de los índices de ubicación del crsp por país y por elección, calculamos un promedio de la ubicación del centro relativo de lo que llamamos sistema político regional (gráfico 2). 
Gráfico 2. Promedio cRSP regional

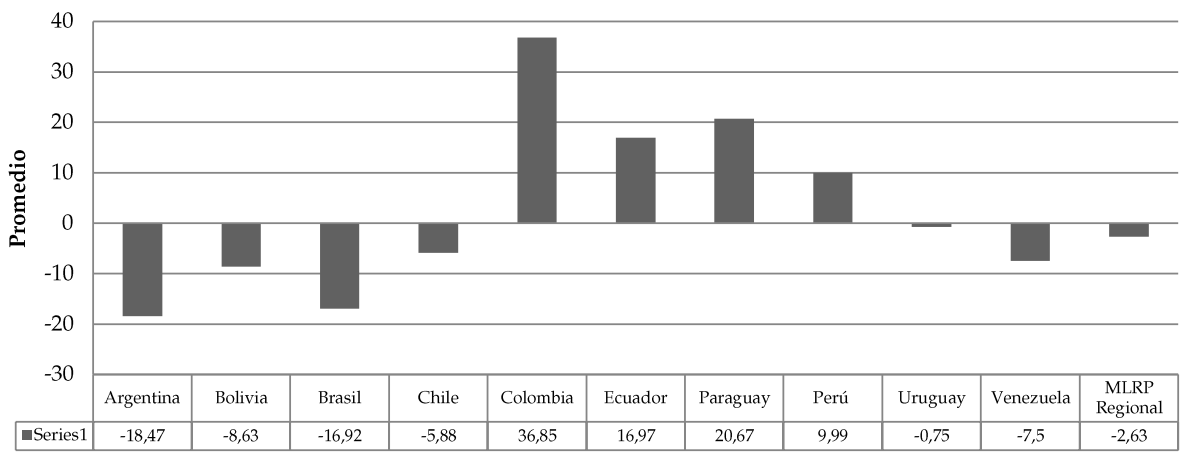

Fuente: elaboración propia a partir de los índices presentados en tabla 1

La fórmula de Coppedge requiere multiplicar por 0,5 los porcentajes de las expresiones políticas ubicadas en la centroderecha y en la centroizquierda. Esta operación intenta equilibrar (como la física cuando calcula la masa) el peso de las expresiones políticas, lo que puede generar resultados anómalos cuando hay múltiples candidatos ubicados en la centroizquierda o en la centroderecha y no existen expresiones políticas de derecha o de izquierda. En esos casos se atenúan las diferencias en los resultados, situación que se comprende cuando se analiza detenidamente la fórmula; escenario que se presentó en la elección presidencial de Chile (2013): la candidata de la Unión Democrática Independiente (UDI), Evelyn Matthei, fue catalogada como una expresión política de derecha, mientras que al otro lado del espectro encontramos una coalición de centro-izquierda (Nueva Mayoría), cuyo porcentaje de votos se multiplicó por 0,5 siguiendo la fórmula. El resultado es que el CRSP chileno permaneció en la centroizquierda, con una distancia menor a la que se pudiera esperar.

Tabla 2. Alineaciones sistemas políticos por elección

\begin{tabular}{|c|c|c|c|c|c|c|}
\hline $\mathrm{N}=45$ & $\begin{array}{c}\text { MLRP } \\
\text { menor a }-41\end{array}$ & $\begin{array}{l}\text { MLRP entre } \\
-40 \text { y }-21\end{array}$ & $\begin{array}{c}\text { MLRP } \\
\text { entre - } 20 \\
\text { y } 0\end{array}$ & $\begin{array}{c}\text { MLRP entre } \\
0 \text { y } 20\end{array}$ & $\begin{array}{l}\text { MLRP entre } \\
21 \text { y } 40\end{array}$ & $\begin{array}{c}\text { MLRP } \\
\text { mayor a } 41\end{array}$ \\
\hline Argentina & & $\begin{array}{c}1999,2007 \text { y } \\
2011\end{array}$ & & 2015 & 2003 & \\
\hline Bolivia & & & $\begin{array}{c}2006,2009 y \\
2014\end{array}$ & 2002 & & \\
\hline Brasil & 2002 & & $\begin{array}{l}2006,2010 y \\
2014\end{array}$ & 1998 & & \\
\hline Chile & & & $\begin{array}{c}1999,2010 y \\
2014\end{array}$ & & & \\
\hline Colombia & & & & 1998 & 2002 & $\begin{array}{c}2006,2009 y \\
2014\end{array}$ \\
\hline
\end{tabular}




\begin{tabular}{|c|c|c|c|c|c|c|}
\hline $\mathrm{N}=45$ & $\begin{array}{c}\text { MLRP } \\
\text { menor a }-41\end{array}$ & $\begin{array}{l}\text { MLRP entre } \\
-40 \text { y }-21\end{array}$ & $\begin{array}{c}\text { MLRP } \\
\text { entre }-20 \\
\text { y } 0 \\
\end{array}$ & $\begin{array}{c}\text { MLRP entre } \\
0 \text { y } 20\end{array}$ & $\begin{array}{c}\text { MLRP entre } \\
21 \text { y } 40\end{array}$ & $\begin{array}{c}\text { MLRP } \\
\text { mayor a } 41\end{array}$ \\
\hline Ecuador & & & 2012 & 2006 y 2009 & 1998,2003 & \\
\hline Paraguay & & & 2013 & 2006 & 1998 y 2003 & \\
\hline Perú & & & 2001 y 2006 & 2011 & 2000 & \\
\hline Uruguay & & & $\begin{array}{c}2004,2009 y \\
2014\end{array}$ & 1999 & & \\
\hline Venezuela & & & $\begin{array}{c}1998,2000, \\
2006,2012 y \\
2013\end{array}$ & & & \\
\hline
\end{tabular}

Fuente: elaboración propia a partir de los índices presentados en la tabla 1

Del análisis de los datos expuestos, se desprenden observaciones relevantes que confirman que las alineaciones de cada sp sudamericano y del sp regional orbitaron alrededor del cRSP. Entre 1998 y 2015, solo el sP brasileño se ubicó a la izquierda en las elecciones de 2002. Este es un punto relevante pues hasta la vanguardia de la marea rosa orbitó en torno al centro relativo. Venezuela y Bolivia constituyen los ejemplos más representativos, observándose en los resultados de todas sus elecciones presidenciales. En Ecuador, como se observa en la tabla 2, el SP se mantuvo en el cuadrante del CRSP, en las elecciones que consagraron a Rafael Correa en 2006, 2009 y 2012. Hallazgos similares, aunque menos novedosos, se presentan en Chile y Uruguay, cuyos sP se mantuvieron estables en torno al centro relativo. En Brasil y Perú se visualiza movilidad del SP: el primero transitó desde el centro relativo hacia la izquierda, mientras que el segundo lo hizo hacia la centroderecha. En ese mismo cuadrante se ubica Colombia, sP cuyo centro político estuvo a contrapelo de la tendencia subregional. Este fue el único caso en el que no se detectó una alineación menor a 0, es decir, en la centroizquierda, en ninguna elección. Incluso Paraguay, país que presenta una alineación similar a Colombia, cruzó la línea de los negativos con el triunfo de Horacio Cartés, representante de la centroderecha. El paisaje argentino dibuja una trayectoria zigzagueante. Los resultados electorales muestran que el sp se ubicó en la centroizquierda (1999), pasó a la centroderecha (2003) y regresó a la centroizquierda (2007 y 2011), para terminar dentro del rango del centro relativo (2015). Los giros muestran que el sP argentino experimentó mayor movilidad y alternancia entre los diversos cuadrantes políticos.

Si interpretamos el conjunto de datos presentados en las tablas, descubrimos que el sp regional se ubicó en una posición de centro durante el período completo. Estos resultados permiten afirmar que el proceso "ascenso de la marea rosa" fue, de alguna manera, sobredimensionado en la literatura de la época. De la misma manera, se sobredimensiona el ascenso de la "marea celeste". El índice promedio del subperíodo $1998-2007$ es 5,13 y para 2008-2015 es 0,433. Estos datos indican que el sp regional orbitó en torno al centro y el índice del último subperíodo, al tomar un valor más próximo a cero, experimentó un desplazamiento - hacia 
la derecha- que lo posiciona en el centro. Asimismo, aclaramos que, en los análisis sobre los giros políticos, se hace más hincapié en el contraste entre los candidatos que gobernaron que en los desplazamientos laterales, como sucedió con el Partido Justicialista, que ha ido progresivamente de izquierda a derecha dependiendo de la coyuntura, internacional o doméstica, o del propio candidato. Por tanto, se puede afirmar que los resultados de la mayoría de las elecciones - 30 sobre 45 - se ubicaron en torno al CRSP.

\section{UNA INTERPRETACIÓN DEL CRSP EN EL ESTUDIO DE LA PE: LOS CASOS DE ARGENTINA Y CHILE}

A partir de los resultados observados en el apartado anterior, escogimos dos países paradigmáticos, Argentina y Chile, para ilustrar empíricamente las orientaciones de sus respectivos sp (gráfico 3). Para analizar las implicancias de la ubicación de los CRSP, se vincula la actuación de los gobiernos a través de sus políticas públicas. En este caso, escogimos la PE y construimos tres dimensiones para relevar su orientación general, a partir de la ubicación de los sp. Ellas son: 1) prioridades geográficas norte versus sur, 2) multilateralismo statuquoista versus multilateralismo revisionista light y 3) regionalismo económico laxo versus regionalismo económico estricto.

Gráfico 3. MLRP por elección por país (1998-2015)

\begin{tabular}{|l|r|r|r|r|r|r|r|r|r|r|r|r|r|r|r|r|r|r|}
\hline & 1998 & 1999 & 2000 & 2001 & 2002 & 2003 & 2004 & 2005 & 2006 & 2007 & 2008 & 2009 & 2010 & 2011 & 2012 & 2013 & 2014 & 2015 \\
\hline Argentina & & $-36,1$ & & & 21,71 & & & $-40,4$ & & & $-43,17$ & & & 5,57 \\
\hline Bolivia & & & & & 15,3 & & & -16 & & & & -17 & & & & & -17 \\
\hline Brasil & 1,85 & & & & $-41,5$ & & & -12 & & & & -17 & & & & -16 \\
\hline Chile & & $-3,4$ & & & & & & $-4,1$ & & & & $-3,4$ & & & & -13 & \\
\hline Colombia & 14,2 & & & & 36 & & & & 43,6 & & & 45,4 & & & 45,1 & \\
\hline Ecuador & 37,7 & & & & 37,1 & & & & 14 & & & 4,58 & & & $-8,4$ & & \\
\hline Paraguay & 33,5 & & & & & 30,4 & & & & & 19,1 & & & & & $-0,2$ & \\
\hline Perú & & & 37,5 & -4 & & & & & -11 & & & & & 17,5 & & & & \\
\hline Uruguay & & 4,87 & & & & & $-2,9$ & & & & & $-2,4$ & & & & & $-2,6$ \\
\hline Venezuela & $-8,1$ & & -10 & & & & & & -13 & & & & & & $-5,4$ & $-0,7$ & \\
\hline
\end{tabular}

Rango de referencia:

Izquierda $\leq-40$
Centro Izquierda -40 a-21
Centro -20 a +20
Centro Derecha 21 a 40
Derecha $\leq 40$

Fuente: elaboración propia a partir de los índices presentados en la tabla 2 


\section{PRIORIDADES GEOGRÁFICAS NORTE VERSUS SUR}

Existe cierto consenso acerca de que los gobiernos "rosas" son proclives a escoger entre sus prioridades a actores geográficos ubicados en el sur global (Hey 1997; Lechini 2009), adoptando posiciones que exhiben distintos grados de hostilidad hacia los actores centrales del sistema internacional: Estados Unidos (EE. UU.), Unión Europea (UE), Banco Mundial (BM) y Fondo Monetario Internacional (FMI). Los alineamientos preferentes con el sur global suelen anclar en la creencia de sus líderes respecto de que los grandes poderes capitalistas aprovechan las asimetrías existentes a su favor, para conservar o profundizar situaciones de dependencia del sur. También sostienen que la asociación con Estados de similar posición tiende a ensanchar sus márgenes de maniobra en PE. Por su parte, los gobiernos "celestes" tienden a ubicar entre sus prioridades externas al conjunto de actores percibidos como hostiles por sus pares de centroizquierda. Los gobiernos "celestes" perciben y seleccionan a sus socios en función de la utilidad que estos posean para alcanzar los objetivos propuestos. Esta posición suele radicar en la creencia de sus líderes respecto de que confrontar sin fundamentos con los grandes actores internacionales perjudicaría la satisfacción de sus intereses, pues podría significar retiro de inversiones, cierre de mercados, dificultades de acceso a capitales, etcétera (Escudé 1992). A partir de los parámetros que fijan estos criterios, analizamos en los dos países su posicionamiento en la elección de prioridades geográficas norte-sur.

En el período en cuestión, Argentina se ubicó en la centroizquierda desde 1999 hasta la elección de 2015. Desde entonces se posicionó en el CRSP. En 1999 asumió el gobierno de la Alianza, ubicado a la centroizquierda del sP, sin embargo, su PE exhibió notables líneas de continuidad con el menemismo. La gestión de Fernando De la Rúa realizó pequeños retoques cosméticos que no cuestionaron ni modificaron sustancialmente las directrices de la PE, pese al incipiente giro político. El canciller Adalberto Rodríguez Giavarini reemplazó la expresión de su antecesor - relaciones carnales- por relaciones maduras y óptimas para caracterizar los vínculos con EE. UU., pero el giro discursivo no modificó el modo de vinculación. Por el contrario, a medida que la crisis argentina se profundizaba, el gobierno necesitó más apoyo de Washington en el FMI. Al igual que durante el menemismo, las relaciones con Europa ocuparon un lugar destacado, observándose algunas modificaciones respecto de la posición argentina en las negociaciones con Gran Bretaña sobre el tema Malvinas, abandonando la "política de seducción". Otro ejemplo en esa dirección fue que Argentina mantuvo la posición adoptada en los noventa respecto del voto de condena a Cuba en la Comisión de Derechos Humanos de la onu (Miranda 2004; Bologna 2006).

Después de una profunda crisis económica y político-institucional, en mayo de 2003 asumió Néstor Kirchner. En líneas generales, la PE de los gobiernos del Frente para la Victoria (FPV) se caracterizó por su corte autonomista, perfil 
latinoamericanista y la definición del interés en términos de desarrollo. De allí se desprende que el alcance de la inserción internacional era selectivo y regional y que sus prioridades fueron las siguientes: MERcosur, Brasil, Chile, Bolivia, China, Venezuela y, en menor medida, EE. uU. y Europa (Lorenzini 2013; Simonoff 2015).

La PE fue utilizada para hacer lobby en el FMI y conseguir apoyos en la negociación de la deuda. Si bien el discurso de Kirchner fue afín y complaciente con los nuevos gobiernos "populistas" de la región - particularmente, Venezuela-, en la práctica el gobierno argentino fue contemplativo y negociador con EE. UU. De hecho, este último país se convirtió en el nexo entre Argentina y el FMI para salir del default, y Venezuela en el prestamista de última instancia (Pereyra Doval 2016). Sin embargo, cabe aclarar que, por la precariedad de la situación del país entre 2003 y 2005, la PE argentina combinó un acercamiento al gobierno norteamericano y la no confrontación con los organismos de crédito especialmente el FMI-, buscando retornar al sistema financiero. Este bienio vio al gobierno argentino en una etapa más contemplativa. Desde 2005, con el pago de la deuda al FMI y la evacuación de las presiones por acordar con el gobierno estadounidense, hubo un endurecimiento en las relaciones con EE. UU. y en el discurso respecto de los organismos de crédito (Llenderrozas 2015).

Otra relación bilateral prioritaria se estableció con Brasil, generando un nuevo acercamiento denominado alianza estratégica, independientemente de la aproximación existente desde mediados de los ochenta y con la creación del MERCosur en los noventa. Sin embargo, esta relación tuvo inconvenientes que no permitieron que la simbiosis superara lo discursivo. En primer lugar, Néstor Kirchner esperaba que su par se comprometiera más con el MERCosur, para sustentar la asociación en la confianza mutua y desplegarla en un conjunto de herramientas jurídicas, políticas y de cooperación dentro del bloque. Es decir, Néstor Kirchner demandó un incremento de la institucionalización del Mercosur, mientras que Lula da Silva optó por ampliar sus opciones de inserción en otros espacios geográficos en lugar de profundizar lo dado.

La cooperación sur-sur (css) se tornó prioritaria en el discurso a partir de la creencia, del gobierno argentino, que los relacionamientos norte-sur eran "intrínsecamente malos", pues concebía a la cooperación fundada en la horizontalidad. En esa dirección se ampliaron las opciones de vinculación y cooperación hacia otros horizontes y con actores no tradicionales de la PEA. Aunque diversos estudios subrayan la cooperación argentina con los continentes africanos y asiáticos, esta se dirigió mayoritariamente hacia Latinoamérica y el Caribe $^{7}$. Todas estas tendencias se mantuvieron en las dos administraciones de Cristina Fernández. 
El frente cambiemos, percibido como un desplazamiento hacia la derecha del SP (Natanson 2017; Vommaro 2017), llegó a la presidencia en 2015 e intentó recuperar los vínculos con EE. UU. y la UE, teniendo en cuenta que estos habían entrado en una situación de stand by desde 2006. Sin embargo, continuó cultivando los vínculos con China, Rusia y los contextos contiguo y regional. Claro que "el ascenso de la marea celeste" puede observarse en los vínculos bilaterales priorizados en la era anterior. Así, en el caso de Venezuela, resulta simple identificar los cambios de la PE argentina ya que la alineación del sP del país caribeño se mantuvo constante. En el caso de Brasil resulta algo más complejo, pues se produjo un giro político y económico a nivel del sP (20132014) que, en cierta forma, acompañó el reacomodamiento argentino.

Por último, percibimos que el gobierno macrista estaría intentando jugar en un esquema de geometría variable (Lechini 2009) desplegando acercamientos hacia un abanico más amplio de actores —individuales y regionales-, aunque no necesariamente pensando en la css como modo de ampliar los márgenes de autonomía, sino como estrategia de diversificación ${ }^{8}$.

En tanto, las características más destacadas de la PE chilena devienen de la adopción del principio de regionalismo abierto que, en líneas generales, guía su inserción internacional desde la redemocratización en 1990 (Rojas Aravena 1997; Van Klaveren 2011). Se destaca el carácter múltiple, diversificado y no excluyente, junto a la búsqueda de relaciones equilibradas como rasgos típicos de una PE con fuerte contenido comercial (Lorenzini 2011; Colacrai 2016). En términos empíricos, se observa en las diversas negociaciones que las distintas administraciones emprendieron y en la vasta red de tratados de libre comercio (TLC), que proyectan una imagen del país como spaghetti bowl conectado con los mercados internacionales (Dirección General de Relaciones Económicas Internacionales [DIRECON] 2009). Cabe subrayar que la PE de Chile exhibe una notoria continuidad en el período analizado y se observa en la ampliación de los mercados como eje ordenador, el mantenimiento de las negociaciones y la selección de sus áreas prioritarias. Ellas son: Europa, Latinoamérica, EE. UU. y Asia-Pacífico, aunque la enumeración no representa un orden taxativo. Tales acciones son convergentes con el regionalismo abierto e ilustran la participación en mesas simultáneas de negociación en el plano global, regional, subregional y extrarregional. Otro de los objetivos consiste en las negociaciones orientadas a acordar reglas de juego para ganar previsibilidad y certidumbre en un ambiente anárquico como el internacional. Del conjunto de acuerdos firmados por Chile, se mencionan los siguientes: Acuerdos de Complementación Económica (ACE) con mercosur, Comunidad Andina de Naciones (CAN), Bolivia, Ecuador y Venezuela; TLC con EE. uU., China, Canadá, Centroamérica, Colombia, European

Aunque la estrategia de diversificación no sea opuesta a la ampliación de los márgenes de autonomía —en algunos casos, la diversificación de los vínculos es una de las opciones que conforman la lógica autonomista-, en el caso del gobierno de Mauricio Macri, la geometría variable no fue pensada como tal. La autonomía que podría generarse con la estrategia de diversificación de socios es residual o un efecto colateral positivo. 
Free Trade Area, Malasia, P4 (Singapur, Nueva Zelanda y Brunei); acuerdos de asociación con la UE y Japón, entre otros. La perdurabilidad de los ejes ordenadores de esta política pública en el largo plazo lo proyecta como socio confiable y nos permite calificar su PE como una política de Estado (Quiroga y Ensignia 2009; Briones y Dockendorff 2015).

El hecho de que Chile haya basado el diseño de su PE en el regionalismo abierto, junto con las cuatro características señaladas, facilita la identificación de su posicionamiento en el eje norte-sur. La preocupación chilena era establecer relaciones con múltiples actores, de modo tal que ello generara cierto equilibrio, evitando una concentración en un único socio para reducir la sensibilidad y vulnerabilidad en un mundo interdependiente. Esto nos muestra que el eje norte-sur no inquietaba a los gobiernos. La Cancillería y la DIRECON procuraron una distribución balanceada de los socios a partir del volumen de los flujos comerciales y las inversiones. El modelo ideal postulaba que el hemisferio occidental, Europa y Asia representaran un 30 \% cada uno, en el esquema económico-comercial de la PE. A la luz de dicho planteamiento, queda claro que la orientación norte-sur no constituyó una preocupación central para las distintas administraciones en la implementación de la PE. No obstante, Chile desarrolló acciones de css durante la primera década del siglo XXI, en sintonía con las tendencias internacionales. Chile concibe a la css como un eje transversal de su PE, que se orienta a lo que los gobiernos han denominado "hacer región", contribuyendo al fortalecimiento institucional, económico-social y científicotécnico en Latinoamérica9.

\section{MULTILATERALISMO STATUQUOISTA VERSUS MULTILATERALISMO REVISIONISTA LIGHT}

Esta dimensión refiere a una constante con respecto a la postura de ambos tipos de gobiernos con respecto a las instancias multilaterales: los rosas suelen ser revisionistas light y los celestes statuquoistas. Esto puede entenderse tomando en cuenta que las posturas gubernamentales se relacionan con el contexto internacional en el que los países se insertan. El cuestionamiento al orden internacional sin alterar sus principios fundamentales - revisionismo light- emergió con más fuerza a comienzos del siglo xxI, cuando un grupo de potencias medias comenzó a exigir un lugar en las paquidérmicas estructuras globales. Estos países se manifestaron de forma contundente en el Consejo de Seguridad (CDS) de la ONU, en la participación en el G-7, en la ponderación de votos en las organizaciones financieras internacionales y en la influencia sobre la agenda de la omc (Nolte 2006). Sin embargo, este revisionismo se limitó a unos pocos discursos altisonantes de algunos gobiernos (entre ellos, el

Ministerio de Relaciones Exteriores de la República de Chile (Agencia de Cooperación Internacional). “Chile y la Cooperación Sur-Sur". Disponible en https://www.cooperacionsursur.org/images/docs/AGCI_Chile_CSS.pdf 
argentino) y a un intento por ascender de categoría, potenciando su jerarquía internacional y acomodándose a las estructuras ya establecidas (entre ellos, el brasileño). Estos últimos, agrupados en coaliciones políticas - BRICsganaron visibilidad y espacio internacional. Con la ampliación de estas redes de contactos internacionales, la influencia de las llamadas potencias medias aumentó notablemente. Por su parte, su actuación en escenarios mundiales es valorada por su capacidad de tender puentes entre países con intereses económicos e ideológicos diferentes. Sin embargo, este ascenso está asociado a que sus intereses son, en general, compatibles con los de las potencias occidentales. Es decir, nunca hubo una agenda contraria al statu quo, sino que, a nivel multilateral, presentaron alguna postura revisionista para integrar el club de los poderosos y no para desmantelarlo.

En Argentina, pese a que el giro a la izquierda se inició en 1999, el gobierno de la Alianza siguió las directrices del gobierno celeste de Carlos Menem, también con respecto al mantenimiento del statu quo en los organismos multilaterales. Argentina no cuestionó las decisiones en el seno de la onU y, en general, acompañó la posición estadounidense. A pesar de abogar por una democratización del CDS, no tuvo una postura definida. Tampoco fueron cuestionados los organismos multilaterales de crédito. De hecho, el país consiguió un "blindaje" de 40000 millones de dólares del FMI.

El "revisionismo" se manifestaría con el gobierno de Néstor Kirchner, quien, habiendo asumido con solo el 22 \% de los votos, rápidamente supo mejorar su imagen supeditando la PE al pago de la deuda con el FMI, concretado en 2005. En efecto, la ruptura con la PE neoliberal se plasmó en el repudio a los organismos financieros a partir de 2005, cuando se suspendieron las visitas examinadoras del FMI y se endureció el discurso. No obstante, el revisionismo de Néstor Kirchner fue más light que el de Cristina Fernández, una dura crítica del orden liberal internacional.

La oleada rosa continuó y se profundizó con las administraciones de Cristina Fernández, quien sugirió, reiteradamente, la reforma de los organismos multilaterales, culpándolos de la crisis argentina de 2001 y, en parte, de la crisis financiera internacional de 2008, a la cual bautizó como "Efecto Jazz". A pesar de sus encendidos discursos, Cristina Fernández se comprometió por escrito, en 2009, a permitir la supervisión del FMI. Pese a este compromiso, volvió a criticar al organismo cuando este sancionó con mociones de censura las mediciones macroeconómicas argentinas.

Con respecto al CDS, el país tuvo importantes fricciones con su socio más cercano - Brasil - por sus reclamos de un asiento como miembro permanente. Sin embargo, cuando se hizo evidente la necesidad de la reforma, en 2005, Argentina volvió a integrar el grupo Unidos por el Consenso, contrario al G-4 y favorable a la creación de diez plazas nuevas no permanentes. 
Con el giro al centro, el gobierno celeste de Mauricio Macri fue elogiado por los organismos financieros por las modificaciones implementadas en materia económica y financiera. Estos halagos y el mutuo acercamiento dan la pauta de un nuevo movimiento que se aproxima al statuquoismo. Ello también se refleja en el marco de la onu, pues en lo que va de su mandato, Mauricio Macri no se pronunció respecto de la modificación del cDs.

Tal como se señaló, Chile condujo su PE en la senda del regionalismo abierto, participando en las negociaciones multilaterales y optando por el bilateralismo para alcanzar el objetivo de ampliación de mercados, como lo evidencian sus más de veinte acuerdos comerciales. Asimismo, cultivó una prudencia combinada con oposiciones moderadas pero firmes frente a situaciones complejas con los grandes poderes. Sin utilizar discursos grandilocuentes: Ricargo Lagos se opuso a la intervención estadounidense en la guerra de Irak (2003), tomando el riesgo de que no se firmara el TLC con EE. UU. Cabe subrayar que el proceso de negociación duró doce años. Durante 2003 se desarrolló la fase en la cual la Embajada de Chile en Washington jugó un rol clave, llevando adelante un conjunto de iniciativas para construir un consenso amplio en el Congreso estadounidense, que permitiera la aprobación del TLC (Bianchi 2003). En ese momento, Chile se desempeñaba como miembro no permanente en el CDS y, en esa coyuntura, se trató el uso de la fuerza por parte de la coalición anglonorteamericana en Irak. Si bien la sesión no se concretó, Chile había hecho pública su posición: no apoyaría la iniciativa norteamericana.

En lo que respecta a la ampliación del CDS, Chile se manifestó a favor y adoptó una posición de equilibrista frente a la puja Argentina-Brasil, ya que ambos países son socios muy importantes, por motivos diferentes. Chile, junto con otros Estados latinoamericanos, apoya la propuesta del G4 "a partir de intereses o compromisos bilaterales" (Sepúlveda y Riquelme 2010: 31). A ello se agrega que Heraldo Muñoz, entonces embajador ante la onU, tuvo un papel importante al haber sido electo como facilitador para el proceso de diálogo en la reforma del cDs. Esta posición muestra un pequeño matiz de revisionismo light en la PE, pues cuestiona alguno de los principios e instituciones sobre los que descansa el orden internacional.

Chile no ha realizado estridentes cuestionamientos a los actores poderosos del sistema internacional (tiene un TLC con EE. UU., otro con China, un acuerdo de asociación con la UE) y varias figuras políticas chilenas ocuparon lugares importantes en instituciones "hegemónicas" como la oEA — José Miguel Insulza- y el FMI — Nicolás Eyzaguirre-. 


\section{POSICIONAMIENTO SOBRE LA INTEGRACIÓN: REGIONALISMO ECONÓMICO LAXO VERSUS REGIONALISMO ECONÓMICO ESTRICTO}

Las visiones de la integración aluden a la preferencia de los actores respecto de la forma en la que los procesos de integración se desarrollan, también al modo en el que deciden su participación. En este sentido, existe cierto consenso con respecto a que los gobiernos "celestes" tienen una visión individualista y liberal, mientras que los "rosas" buscan formas más profundas, con tintes solidarios.

El regionalismo económico laxo (REL) es aquel que permite que los Estados diversifiquen sus vínculos comerciales vía TLC O ACE con escasas restricciones, optando por formas menos comprometidas de integración como las zonas de libre comercio (ZLC). El regionalismo económico estricto (REE), por su parte, refiere a formas de integración que demandan mayores compromisos y que recortan márgenes de acción a los Estados miembros en negociaciones con terceros. Las uniones aduaneras (UA) representan un límite a la política comercial pues las negociaciones tienen que hacerse en conjunto con el bloque. De acuerdo con lo establecido supra, los gobiernos "celestes" preferirían esquemas de REL, mientras que los "rosas" optarían por un REE.

Argentina fue "adaptando" su estrategia de acuerdo con el gobierno de turno y atravesó encendidos debates internos - Cancillería versus Ministerio de Economía- en el camino. Chile combina su pertenencia sudamericana con una estrategia de inserción múltiple cuyas características permanecen sin grandes variaciones.

Argentina exhibió líneas de continuidad en su PE durante las administraciones Menem-De la Rúa. Pese a haber optado por hacer del MERcosur una UA, el interés argentino estaba definido en términos de crecimiento económico, lo que se aproxima a un regionalismo económico, más bien laxo. Por tanto, a excepción de la puesta en práctica del arancel externo común, la coordinación de políticas y las negociaciones conjuntas no registraron grades avances. La integración regional fue utilizada como instrumento de política eficaz para intensificar la liberalización comercial y reducir el promedio arancelario. La apertura comercial produjo los beneficios derivados de una mayor competencia, una disminución de los costos de los insumos y la ampliación de las posibilidades de consumo, aunque introdujo nuevas dificultades, sufridas, mayoritariamente, por Argentina. Estas supusieron costos fiscales y políticos, déficit comercial, crecimiento del desempleo debido a las modificaciones en las coaliciones económicas nacionales. Así, a pesar de que el gobierno de Fernando De la Rúa buscó diferenciarse del anterior, estuvo fuertemente condicionado por la delicada situación económica y financiera heredada, que se agravó por las débiles y erráticas políticas implementadas. Por esto, tanto Fernando De la Rúa como su sucesor, Eduardo Duhalde, no tuvieron oportunidad de desarrollar e implementar lineamientos claros respecto de la integración regional. 
Con relación al Área de Libre Comercio de las Américas (ALCA), Argentina exhibió una postura ambivalente. Durante las dos administraciones de Carlos Menem y sobre la base del espíritu no excluyente del REL, mostró interés en iniciar negociaciones, aun vulnerando el formato 4+1 del MERCOSUR. El gobierno de Fernando De la Rúa no descartó la negociación del ALCA, sin embargo, acordó con Brasil un esquema de negociación conjunto, acercándose al REE.

Este giro siguió profundizándose con el gobierno de Néstor Kirchner, en el cual se analizó la condición periférica del Cono Sur con un criterio optimista, en la medida en que los países de la región buscaron asociarse para manejar conjuntamente situaciones adversas. Los espacios multilaterales con posturas comunes, la alternativa de compartir situaciones regionales y los procesos de integración, ocuparon un lugar decisivo en la PE. Por tanto, entre sus rasgos destacados subrayamos la opción por pensar la reinserción internacional argentina desde el mercosur. Con respecto al ALCA, Argentina fue sede de la Cuarta Cumbre, aunque la posición exhibida fue contraria a su conformación, priorizándose esquemas regionales como UNASUR. Cristina Fernández acentuó la alianza estratégica argentino-brasileña, la cual se erigió como eje articulador de la reactivación del MERCOSUR y UNASUR, bajo los parámetros del regionalismo posliberal (Da Motta Veiga y Ríos 2007; Sanahuja 2012). De acuerdo con las características de este último, las cuestiones políticas, ideológicas y sociales constituyeron el centro de atención de la agenda y, de alguna manera, reemplazaron el énfasis en la dimensión comercial, lo que, paradójicamente, acentuó la opción por el REE.

Durante la administración Mauricio Macri se prioriza la necesidad de resolver aquellas barreras que obstaculizaban el comercio. Este giro en la concepción de la integración es convergente con las características del REL, que propicia nuevos acuerdos de apertura y liberalización comercial a través de la construcción de nuevas normas. Teniendo en cuenta la importancia asignada a la cuestión comercial y su regulación, así como también las necesidades diagnosticadas por este gobierno, se observa un marcado interés en trabajar con sus socios del MERCOSUR para lograr la inserción de Argentina y sus vecinos en otras iniciativas como la Alianza del Pacífico (AP). Esta situación contrasta con la percepción de buena parte de los gobiernos rosas, que consideraban que la AP era una iniciativa al servicio de los intereses estadounidenses. En consonancia, Argentina ingresó como observador a la AP, lo cual se constituye en una oportunidad de negocios y, según declaraciones de otros Estados latinoamericanos, es visualizada positivamente pues posibilitaría una negociación AP-MERCOSUR.

Por su parte, en Chile predominó, como característica distintiva de su PE, el carácter múltiple observado en la red de ACE y TLC suscritos desde los noventa. Las características más relevantes del diseño de la PE devienen del regionalismo abierto — que se corresponde con la versión laxa- y se destacan: su carácter múltiple, diversificado y no excluyente, a partir de una ecuación racional entre autonomía y pragmatismo. 
Así, se sostienen comportamientos muy diferenciados. Con respecto a las negociaciones para la conformación del ALCA, Chile siempre tuvo una posición favorable, coherente con los ejes ordenadores de su PE. Cabe recordar que fue candidato para sumarse al NAFTA. Cuando las negociaciones con EE. UU. se estancaron, Eduardo Frei Ruiz-Tagle inició negociaciones con Canadá (1996) y México (1998 y 2006). La pieza que faltaba para completar la inserción en Norteamérica era el TLC con EE. UU., firmado en 2003. Cuando las negociaciones hemisféricas se aletargaron, Chile trabajó con Centroamérica, firmando un TLC en 1999. Además, se había asociado al MERCosur (1996) y a la CAN (2006).

Por otro lado, Chile se vinculó de manera dinámica y activa con Asia-Pacífico. Su ubicación geográfica y los $4500 \mathrm{~km}$ de costas sobre ese océano contribuyeron para que este Estado mirara aquella región. La vinculación más reciente fue incorporada a la estrategia de inserción regional gradual y pragmática, que combinó multi y bilateralismo. Ambos niveles se evidenciaron en la asociación paulatina en los foros de cooperación - PBEC, PECC, APEC - y en el desarrollo de vínculos Estado-Estado como los TLC, acuerdos de alcance parcial y de asociación con Corea del Sur, China, India y Japón, respectivamente.

Los numerosos acuerdos que vinculan comercialmente a Chile con un amplio abanico de actores es un claro ejemplo de REL que posibilita la convivencia de alineamientos múltiples, flexibles y no excluyentes. Ejemplos en esa dirección son su incorporación solo como Estado asociado al MERCOSUR y a la CAN, reservándose un amplio margen de gestión en su política comercial externa. En suma, Chile conjugó su pertenencia a la región de la cual forma parte con algunas ambivalencias-y se insertó en otras regiones, diversificando sus vínculos.

\section{VII.REFLEXIONES FINALES}

Durante la primera década del siglo xxI, el eje del debate regional se centró en el denominado "giro a la izquierda". Sin embargo, las categorías izquierda-derecha por sí mismas nos dicen poco acerca de la realidad. En consonancia, Coppedge (2000) explica que las críticas de los múltiples actores políticos al Consenso de Washington, las políticas neoliberales implementadas y el deterioro de los indicadores sociales provocaron el desplazamiento hacia la centroizquierda del centro ideológico sudamericano. Tras una década de gobiernos rosas, con resultados dispares y cuestionados, triunfó en las urnas una oleada de gobiernos asociados a la marea celeste. A través de la utilización de la metodología de Coppedge, afirmamos que el ascenso de la marea rosa de principios de siglo provocó un desplazamiento del CRSP regional y que dicho movimiento condujo al reconocimiento de lo que se hizo bien en el pasado reciente, dejando en claro qué aspectos eran necesarios corregir. Sobre los aciertos y desaciertos de los gobiernos rosas se montaron las plataformas electorales de los partidos de oposición que, finalmente, ganarían las elecciones en la región. Esto generó un 
nuevo desplazamiento de los CRSP que, según el país, se ubicaría en el centro o en la centroderecha.

Desde un punto de vista simbólico, la muerte de Hugo Chávez representó el inicio del fin de los progresismos latinoamericanos. Este nuevo proceso encontró en los liderazgos de Juan Manuel Santos, Sebastián Piñera, Pedro Pablo Kuczynski, Horacio Cartés y Mauricio Macri los bastiones de una representación política más liberal. A ello se sumaron hechos que debilitaron la continuidad de la marea rosa y generaron el ascenso de la marea celeste: el triunfo de Mauricio Macri (2015), la victoria de la oposición en las elecciones parlamentaria venezolanas (2015), la derrota de Evo Morales en el referendo para otro ciclo de gobierno (2016) y el juicio político y posterior destitución de Dilma Rousseff (2016). Tales acontecimientos parecerían reflejar una parcial pérdida de apoyo popular, que se traduce en un menor grado de legitimidad que la que tuvieron los gobiernos rosas.

A partir del cálculo del CRsP regional, se observan tres conclusiones esenciales. La primera es que, durante el período analizado, aunque el centro relativo se haya movido a los laterales, siempre estuvo en el rango de las posiciones de centro. La segunda es que, en las elecciones realizadas, los sP más estables de la región (Chile y Uruguay) permanecieron inclinados a la izquierda, siendo que el índice que mide esa variación experimentó pequeños movimientos. La tercera es que en las elecciones realizadas en los países más "politizados" (Argentina, Perú y Paraguay) se observan cambios en el signo de la polarización.

A la luz del argumento del CRSP, la PE fue analizada a partir de la construcción de tres dimensiones: 1) prioridades geográficas norte-sur, 2) multilateralismo statuquoista versus multilateralismo revisionista light y 3) regionalismo económico laxo versus regionalismo económico estricto. En el caso argentino, el primer giro político (1999-2003) fue en términos discursivos y no tuvo un correlato empírico ni en el diseño ni en la ejecución de la PE. Por el contrario, continuó los lineamientos centrales de la administración anterior, ubicada en la centroderecha. A partir de 2003, el triunfo del FPV y sus tres administraciones -Néstor Kirchner y las dos de Cristina Fernández- se asoció a los gobiernos de la marea rosa. En esta ocasión, la retórica fue acompañada con acciones concretas que convergieron con las posiciones tradicionales asociadas a cada una de las tres dimensiones: preferencia por socios del sur global, multilateralismo revisionista light y regionalismo económico estricto. En noviembre de 2015 observamos un nuevo giro del CRSP, que ubica al gobierno en el centro, exhibiendo un desplazamiento hacia la derecha, motivo por el cual se lo asocia a la marea celeste. En este caso se observa un cambio discursivo más moderado y menos confrontacionista que el anterior, cuyo análisis permite ubicar la gestión de la PE en el centro del sP, aunque la oposición los coloque en la derecha. Ello supone el mantenimiento de algunas directrices de la PE: el Mercosur como espacio desde el cual se piensa y se proyecta la inserción internacional, la prioridad a los vínculos con China y Bolivia; así como también algunos cambios: la incorporación como observador a la AP, la recomposición 
de los vínculos con EE. UU., FMI y UE, el enfriamiento y distanciamiento en la relación con Venezuela. Si bien hubo acciones que se identifican con posiciones de derecha, también existen otras que coinciden con los posicionamientos atribuidos a la izquierda en las tres dimensiones. Así, los desplazamientos en la posición del CRSP redundaron en cambios en la PE, aunque conservan el carácter "relativo" del argumento de Coppedge.

El caso de Chile se transforma en la excepción que confirma la regla, pues se trata de un país gobernado durante una década (1990-2010) por una coalición de centroizquierda cuyas posiciones en materia de PE no se condicen con la mayoría de los gobiernos rosas. Se observa que los actores políticos de centroizquierda, así como los de centroderecha, presentan anomalías frente al libreto oficial: las suposiciones generalizadas sobre las creencias y acciones típicas de actores políticos de centroizquierda y centroderecha, respectivamente. Estas singularidades pueden advertirse, en algunos casos, cuando se toma como referencia una PE que se constituye como política de Estado. La permanencia del SP chileno en el centro relativo contribuyó a la continuidad de las líneas directrices de la PE en el período 1998-2015. Sus líderes fueron capaces de articular los consensos de los distintos sectores, cumpliendo así uno de los objetivos centrales, formulado en 1990: hacer de la PE una verdadera política de Estado.

\section{REFERENCIAS}

Bologna, Alfredo Bruno (ed.). 2006. La politica exterior del gobierno de Kirchner. Rosario: UNR Editora-CERIR.

Actis, Esteban, María Elena Lorenzini y Julieta Zelicovich. 2016. "Modelo de desarrollo y estrategia de inserción. Claves para la interpretación de su relación". En Modelos de desarrollo e inserción internacional. Aportes para el análisis de la política exterior argentina desde la redemocratización 1983-2011, editado por Anabella Busso. Rosario: unR Editora, 15-30.

Bianchi, Andrés. 2003. “El Tratado de Libre Comercio Chile-Estados Unidos. Un caso de diplomacia moderna". Diplomacia 100: 27-43.

Briones, Sebastián y Andrés Dockendorff. 2015. "Continuidad y cambio en la política exterior chilena en el gobierno de Piñera". Estudios Internacionales 47 (180): 115-138.

Busso, Anabella (ed.). 2016. Modelos de desarrollo e inserción internacional. Aportes para el análisis de la política exterior argentina desde la redemocratización (1983-2011). Rosario: unR Editora.

Castañeda, Jorge. 2006, mayo-junio. "Latin America's Left Turn". Recuperado el 3 de abril de 2018 de http://www.foreignaffairs.com/articles/61702/jorge-g-castaneda/latin-americas-left-turn

Clemente Batalla, Isabel. 2007. "Inserción internacional desde la región". Cuadernos del CLAEH 94-95 (30): 11-33.

Colacrai, Miryam. 2016. "La relación bilateral Argentina-Chile en clave política 2010-2015. Continuidades y matices con cambios de gobiernos". Estudios Internacionales 48 (183): 9-37.

Coppedge, Michael. 1997. "District Magnitude, Economic Performance, and Party-System Fragmentation in Five Latin American Countries". Comparative Political Studies 30 (2): 156-185. 
Coppedge, Michael. 2000. “La diversidad dinámica de los sistemas de partidos latinoamericanos". POSTData 6: 109-134.

Curia, Eduardo. 2011. El modelo de desarrollo en Argentina. Los riesgos de una dinámica pendular. Buenos Aires: Fondo de Cultura Económica-FCE.

Da Motta Veiga, Pedro y Sandra Ríos. 2007. “O Regionalismo pós-liberal, na América do Sul: origens, iniciativas e dilemas". CEPAL 82: 1-48.

Dirección General de Cooperación Internacional (DGCIN). 2015. “Lineamientos para la Cooperación Sur-Sur (2013-2015)". Recuperado de http:/ / www.cooperacionar.gob.ar

Dirección General de Relaciones Económicas Internacionales (2009). Chile: 20 años de ne gociaciones comerciales. Santiago: DIRECON.

Doeser, Fredrik. 2011. "Domestic Politics and Foreign Policy Change in Small States: The Fall of the Danish 'Footnote Policy'". Cooperation and Conflict 46 (2): 222-241.

Doyle, Michael. 1986. "Liberalism and World Politics". The American Political Science Review 80 (4): 1151-1169.

Escudé, Carlos. 1992. El realismo periférico. Fundamento para la nueva política exterior argentina. Buenos Aires: Planeta.

George, Alexander. 1993. Bridging the Gap: Theory and Practice in Foreign Policy. Washington: United States Institute of Peace.

Giordano, Verónica. 2014. "¿Qué hay de nuevo en las "nuevas derechas"?". Nueva Sociedad 254: 46-56.

Goldstein, Judith y Robert Keohane. 1993. Ideas and Foreign Policy. Ithaca, NY: Cornell University Press.

Grasa, Rafael. 1993. "Las organizaciones internacionales y los nuevos desafíos globales". Papers. Revista de Sociología 41: 55-84.

Hermann, Charles. 1990. “Changing Course: When Governments Choose to Redirect Foreign Policy". International Studies Quarterly 34 (1): 3-21.

Hermann, Margaret y Charles Hermann. 1989. "Who Makes Foreign Policy Decisions and How: An Empirical Inquiry". International Studies Quarterly 33 (4): 361-387.

Hermann, Margaret. 2001. "How Decision Units Shape Foreign Policy: A Theoretical Framework". International Studies Review 3 (2): 47-81.

Hey, Jeanne. 1997. "Three Building Blocks of a Theory of Latin American Foreign Policy". Third World Quarterly 18 (4): 631-657.

Holsti, Kalevi. 1982. Why Nations Realign: Foreign Policy in the Postwar World. Boston: Allen \& Unwin.

Hudson, Valerie. 2005. "Foreign Policy Analysis. Actor-Specific Theory and the Ground of International Relations". Foreign Policy Analysis 1 (1): 1-30.

Keohane, Robert. 1988. Después de la hegemonía. Cooperación y discordia en la política económica mundial. Buenos Aires: Grupo Editor Latinoamericano.

Lechini, Gladys. 2009. “La cooperación sur-sur y la búsqueda de autonomía en América Latina. ¿Mito o realidad?". Relaciones Internacionales 12: 55-81.

Llenderrozas, Elsa. 2015. “Elecciones presidenciales y política externa: hacia la complementariedad de espacios políticos no excluyentes". Revista de la Facultad de Ciencias Sociales 89: 60-65.

Lorenzini, María Elena. 2011. Política exterior, alianzas estratégicas y energía en América Latina. Las relaciones argentino-chilenas bajo la lupa. Rosario: Homo Sapiens.

Lorenzini, María Elena. 2013. "Las políticas exteriores de Argentina y Venezuela en el siglo xxI. Una primera aproximación al análisis comparado (2003-2011)". Reflexión Política 15: 38-52.

Miranda, Roberto. 2001. "El cambio externo y las estrategias internacionales de la Argentina". Relaciones Internacionales 10 (21): 169-195.

Miranda, Roberto. 2004. "Imagen de cambio. Los primeros meses de la política internacional del gobierno de Néstor Kirchner". En Anuario 2004 de Relaciones Internacionales, editado por Instituto de Relaciones Internacionales. La Plata: Universidad de la Plata, 1-9. 
Natanson, José. 2017. "La "ola amarilla" en Argentina. Reconfiguraciones tras el triunfo de Cambiemos". Nueva Sociedad 272: 4-12.

Nolte, Detlef. 2006. "Potencias regionales en la política internacional: conceptos y enfoques de análisis". GIGA Working Papers 30.

O’Neil, Shannon. 2016. “Latin America's Populist Hangover". Foreign Affairs 95 (6): 31-38.

Panizza, Francisco. 2006. "La marea rosa". Correo Americano 8: 5-24.

Pereyra Doval, Gisela. 2016. "Modelos de desarrollo y política exterior como trasfondo de la cooperación sur-sur en Argentina y Brasil en el siglo xxI". En Poderes emergentes y cooperación sur-sur. Perspectivas desde el sur global, editado por Gladys Lechini y Clarisa Giaccaglia. Rosario: uNR Editora, 70-85.

Puig, Juan Carlos. 1984. "La política exterior Argentina. Incongruencia epidérmica y coherencia estructural". En América Latina. Políticas exteriores comparadas, editado por Juan Carlos Puig, Tomo I. Buenos Aires: GEL, 91-169.

Quiroga, Yesko y Jaime Ensignia. 2009. Chile en la Concertación (1990-2010). Una mirada crítica, balances y perspectivas. Santiago: Friedrich Ebert Stiftung.

Risse-Kappen, Thomas. 1994. "Ideas Do Not Float Freely: Transnational Coalitions, Domestic Structures and the End of Cold War". International Organization 48 (2): 185-214.

Rojas Aravena, Francisco. 1997. "Chile: cambio político e inserción internacional 1964-1997". Estudios Internacionales 30 (119-120): 376-407.

Rosatti, Jerel. 2000. "The Power of Human Cognition in the Study of World Politics". International Studies Review 2 (3): 45-75.

Rosenau, James. 1995. "Signals, Signposts and Symptoms: Interpreting Change and Anomalies in World Politics". European Journal of International Affairs 1 (1): 113-122.

Sanahuja, José A. 2012. “Regionalismo posliberal y multilateralismo en Sudámerica: el caso de Unasur". Anuario de Integración 9: 19-71.

Schmidt, Brian. 2008. "The Primacy of National Security". En Foreign Policy. Theories, Actors, Cases, editado por Steve Smith, Amelia Hadfiel y Timothy Dunne. Oxford: Oxford University Press, 155-170.

Sepúlveda, Juan Pedro y Jorge Riquelme. 2010. “La reforma del Consejo de Seguridad. Una mirada desde América Latina". Nueva Sociedad 230: 23-36.

Simonoff, Alejandro. 2015. "Diez años de política exterior argentina". Relaciones Internacionales 11 (22): 1-24.

Van Klaveren, Alberto. 2011. “La política exterior de Chile durante los gobiernos de la Concertación (1990-2010)". Estudios Internacionales 44 (169): 155-172.

Vommaro, Gabriel. 2017. "La centroderecha y el "cambio cultural" argentino". Nueva Sociedad 270: 4-13.

Waltz, Kenneth N. 1988. Teoría de la política internacional. Buenos Aires: Grupo Editor Latinoamericano.

Waltz, Kenneth N. 2000. "Structural Realism after the Cold War". International Security 25 (1): 5-41.

Wohlforth, William. 2008. "Realism and Foreign Policy". En Foreign Policy. Theories, Actors, Cases, editado por Steve Smith, Amelia Hadfiel y Timothy Dunne. Oxford: Oxford University Press, 31-48.

Recibido: 31 de marzo de 2018

Aceptado: 15 de noviembre de 2018 
María Elena Lorenzini. Doctora (Ph.D.) en Relaciones Internacionales por la Universidad Nacional de Rosario (UNR) y Magíster en Integración y Cooperación Internacional por el Centro de Estudios en Relaciones Internacionales de Rosario. Es investigadora adjunta del CONICET y profesora titular en la cátedra Teoría de las Relaciones Internacionales en la Facultad de Ciencia Política y Relaciones Internacionales de la UNR. Su área de especialización es la política exterior sudamericana. Su trabajo se focaliza en las alianzas estratégicas en la política exterior argentina en comparación con Chile y Venezuela. Ha publicado sobre sus temas de investigación en Foreign Affairs Latinoamérica, Íconos, ConFines, entre otras, además de un libro de autoría individual y múltiples capítulos de libros. Ha impartido clases de posgrado en la UNR, la Universidad Nacional de Brasilia, FLAcso-Ecuador y el CERIR. Correo electrónico: melorenzini@gmail.com

Gisela Pereyra Doval. Doctora (Ph.D.) en Relaciones Internacionales por la Universidad Nacional de Rosario. Es investigadora adjunta del conicET y profesora adjunta en la cátedra Problemática de las Relaciones Internacionales en la Facultad de Ciencia Política y Relaciones Internacionales de la UNR. Su área de especialización es la política exterior sudamericana. Su trabajo se focaliza en las percepciones y la dimensión identitaria de las políticas exteriores de Argentina y Brasil. Ha publicado sobre sus temas de investigación en India Quarterly, Estudos Internacionais, Civilizar, Apuntes, entre otras, además de múltiples capítulos de libros. Ha impartido clases de posgrado en la UnR, la Universidad Nacional de Brasilia y la Universidad Católica de Santa Fe. Correo electrónico: gpdoval@gmail.com 